\title{
Studie 2: Normwerte der Berufswahlkompetenz: Eine diagnostische Chance zur individuellen Förderung?
}

\subsection{Diagnostik im Rahmen schulischer Unterstützung beruflicher Orientierung}

Die Feststellung persönlicher Bedarfe stellt die Grundlage individualisierter, passgenauer Unterstützung in der berufswahlrelevanten Entwicklung Jugendlicher dar. Neben persönlichen und sozioökonomischen Merkmalen der Heranwachsenden (Institut für Demoskopie Allensbach, 2014) beeinflussen ihre bereits bestehenden berufswahlrelevanten Kompetenzen, also die Gesamtheit ihrer „kognitiven Fähigkeiten und Fertigkeiten [...] sowie die damit verbundenen motivationalen, volitionalen und sozialen Bereitschaften“ (Weinert, 2001b, S. 27 f.) zur Problemlösung ihre weiteren Berufsorientierungsbedarfe. Dabei entfalten individualisierte Angebote eine größere Wirksamkeit, während für standardisierte Maßnahmen beruflicher Orientierung in Abhängigkeit der Zielgruppe nur geringe bis keine Effekte nachgewiesen werden können (Ohlemann \& Ittel, 2017; Whiston, Brecheisen \& Stephens, 2003). So scheint es eine logische Schlussfolgerung, dass die schulische Unterstützung beruflicher Orientierung (BO) durch passgenaue Angebote stärker an den individuellen Bedarfen ausgerichtet werden muss. Bei der Umsetzung individualisierter BO stehen die Schulen wie eingangs bereits ausgeführt jedoch vor einer Reihe an Herausforderungen: Neben finanziellen und personellen Restriktionen bestehen zeitliche und organisatorische

Diese empirische Studie wurde 2018 in ähnlicher Form veröffentlicht und kann wie folgt zitiert werden: Ohlemann, S. \& Ittel, A. (2018). Normwerte der Berufswahlkompetenz: Eine diagnostische Chance zur individuellen Förderung? In E. Wittmann, D. Frommberger \& B. Ziegler (Hrsg.), Jahrbuch der berufs- und wirtschaftspädagogischen Forschung 2018 (S. 111-123). Opladen: Barbara Budrich. 
Grenzen, die der Auswertung komplexer Testverfahren zur Diagnostik individueller Entwicklungsstände entgegenstehen (Ohlemann et al., 2016). Daraus lässt sich die Notwendigkeit eines einfachen diagnostischen Instruments für Schulen zur Bestimmung der individuellen Ausgangslage Jugendlicher ableiten. Denkbar wäre ein übergeordneter diagnostischer Kernwert der beruflichen Orientierung, der ähnlich wie bei der Messung der Intelligenzleistung verschiedene Subskalen zusammenfasst. Schulen müssten anhand dieses einzelnen Kernwerts den Entwicklungsstand der Jugendlichen leicht einordnen und gleichzeitig aus diesem Rückschlüsse auf die verschiedenen Unterdimensionen ziehen können, um daraufhin die individuellen Bedarfe adressieren zu können. Die folgende Studie widmet sich dementsprechend der Frage, inwiefern die Konstruktion eines einzelnen komplexitätsreduzierenden Normwerts zur Diagnostik von Berufswahlkompetenzen möglich ist.

\subsection{Theoretische Einbettung berufswahlrelevanter Diagnostik}

\section{Normierungen, diagnostische Normwerte und Instrumente}

Normwerte und normorientierte Tests finden eine breite Anwendung u. a. in der psychopathologischen Diagnostik (Payk, 2015), um unter Bezugnahme auf eine relevante, repräsentative Gruppe (Normstichprobe) Merkmalsausprägungen eines Individuums einzuordnen (Bühner, 2011). Die IQ-Norm wird beispielsweise zur zeitnahen Quantifizierung und Einschätzung einer intellektuellen Einzelleistung eines Menschen verwendet. Sie umfasst dabei Teilfähigkeiten, wie logisches Denken und Gedächtnisleistung (vgl. Bühner, 2011; D. H. Rost, 2009). Die Vorteile von Normierungen, durch reliable Vergleichswerte eine schnelle Orientierung und Unterstützung in der Diagnostik zu erhalten, scheinen offensichtlich. Gleichzeitig sollte der Einsatz von Normwerten kritisch und mit Bedacht geschehen. Ihre sinnhafte Interpretation hängt aufgrund kultureller, soziodemografischer und situativer Unterschiede zwischen Testpersonen stark von der Bezugnahme zur passenden Vergleichsgruppe ab (vgl. Scharfetter, 1996). Ein Normwert muss dementsprechend auch Rückschlüsse auf ihm zugrundeliegende Teilfacetten zulassen. Zudem bleibt zu bedenken, dass Normwerte statistische Richtwerte sind, die im gesellschaftlichen und fachlichen Konsens gesetzt werden (vgl. Scharfetter, 1996).

Im berufsorientierenden Bereich reicht das Spektrum diagnostischer Instrumente von Feststellungsverfahren zu berufswahlrelevanten Interessen und Fähigkeiten hin zu Berufsorientierungstests, die anhand berufsfeldtypischer Aufgaben die Eignung der Heranwachsenden hinsichtlich einer Auswahl an Berufen analysieren. Jedoch 
werden die Ergebnisse standardisierter (Online-)Tests oft nicht in einer professionellen Beratung reflektiert, um sie einerseits in Bezug zu den Lebensentwürfen der jungen Menschen zu setzen (Thomas, 2013) und andererseits als „Baustein“ (Kanning, 2013, S. 249) in ein ganzheitliches Konzept beruflicher Orientierung zu integrieren. Die beschriebenen Instrumente fokussieren die Entwicklung einer oder mehrerer spezifischer Kompetenzen, ein diagnostisches Verfahren zur Feststellung des individuellen Entwicklungsstands, auf dem aufbauend Bedarfe für weitere Maßnahmen ermittelt werden können, fehlte jedoch bisher. Kaak et al. (2013) haben das soweit einzige theoriegeleitete Instrument im deutschsprachigen Raum entwickelt, das Entwicklungsstände berufswahlrelevanter Kompetenzen erfasst. Das zugrundeliegende theoretische Modell der Berufswahlkompetenz von Driesel-Lange et al. (2010) sowie der diagnostische Fragebogen, auf dem die vorgelegte Studie basiert, wurden in Kapitel 6 detailliert beschrieben. Bezugnehmend auf die Fragestellung soll zudem hier auf die Komplexität der händischen Fragebogenauswertung, die durch das pädagogische Schulpersonal vorgenommen werden muss, hingewiesen werden (vgl. Lipowski et al., 2015b).

\subsection{Durchführung der Studie}

\section{Fragestellung und Hypothesen}

Mit dem Ziel schulinterner individualisierter Maßnahmen-Attribution stellt sich die Frage, wie der Berufswahlkompetenzstand Jugendlicher am einfachsten in Schulen diagnostiziert werden könnte. Verschiedene Studien mit clusteranalytischen Verfahren (Kaak et al., 2015; Ohlemann \& Driesel-Lange, 2017) sowie Vorstudien anhand latenter Profilanalysen konnten in diesem Zusammenhang Entwicklungsprofile der Berufswahlkompetenz darstellen. Die identifizierten Profile folgten jeweils ähnlichen Entwicklungsmustern, bei denen die zwölf BWK-Facetten innerhalb eines Profils immer in ähnlicher Relation zueinanderstanden. Diese Profile unterschieden sich also vor allem in ihrem Gesamtniveau. Daraus leitet sich die Frage ab, inwiefern die zwölf BWK-Facetten sinnvoll zu einem Normwert, dem BWK-Kernwert, analog zu anderen Normwerten zusammengefasst und dadurch in ihrer Komplexität reduziert werden können, um individuelle Bestandsaufnahmen aller Lernenden im Rahmen der verfügbaren zeitlichen und personellen Ressourcen zu ermöglichen.

Drei Hypothesen ergeben sich:

(1) Analog zu den oben genannten Analysen, bei denen die BWK-Facetten nicht aufsummiert wurden, lassen sich auch mittels des BWK-Kernwerts Gruppen mit 
ähnlichen Entwicklungsmustern für die zwölf Berufswahlkompetenzfacetten identifizieren.

(2) Wie bei den Profilanalysen lassen sich Gruppen bezogen auf die BWK-Facetten klar voneinander differenzieren.

(3) Der BWK-Kernwert lässt Rückschlüsse auf den Entwicklungsstand einzelner BWK-Facetten zu.

\section{Datengrundlage}

Die vorgelegte Studie basiert auf einer Stichprobe von 1065 Jugendlichen (weiblich: $n=537 ; 51 \%$ ) aus drei längsschnittlichen Studien ${ }^{1}$, die im Einzelnen in Abschnitt 8.1 vorgestellt wurden. Von allen Teilnehmenden besuchten 454 (43\%) eine Berufsschule in Nordrhein-Westfalen (NRW). 310 Jugendliche lernten in der Jahrgangsstufe neun bzw. zehn einer Integrierten Sekundarschule in Berlin. 134 bzw. 167 Teilnehmende besuchten die zehnte Klasse an einer Gesamtschule bzw. einem Gymnasium in NRW. Der Anteil der Schülerinnen betrug in den Substichproben zwischen $44 \%-58 \%$. Die Befragungen im Klassenverband fanden während des regulären Unterrichts statt.

\section{Instrumente \& Methode}

Zur Erhebung der Berufswahlkompetenz wurde der Fragebogen zur Messung der Berufswahlkompetenz von Kaak et al. (2013) verwendet, der in Abschnitt $6.2 \mathrm{im}$ Detail erläutert wurde. Die Jugendlichen schätzten ihren persönlichen Entwicklungsstand hinsichtlich der zwölf Berufswahlkompetenzfacetten anhand von 90 Items im vierstufigen Likert-Format ( $1=$ trifft überhaupt nicht zu, $4=$ trifft voll zu) jeweils selbst ein. Cronbachs Alpha lag für die zwölf BWK-Facetten zwischen 67 (Planungskompetenz) und .89 (Exploration) wie die Tabelle $10.1 \mathrm{im}$ Detail zeigt.

Um die Hypothese des Normwerts, der die zwölf BWK-Facetten widerspiegelt, testen zu können, wurde der Mittelwert der zwölf BWK-Facetten gebildet (Tabelle 10.2). Er wird folgend als BWK-Kernwert bezeichnet.

Die Teilnehmenden wurden nach Höhe ihres individuellen BWK-Kernwerts in einer Rangreihe angeordnet und dann gemäß ihrem Prozentrang in eine von neun Staninegruppen eingruppiert (Tent \& Stelzl, 1993; vgl. auch Bühner, 2011). Stanine, kurz für standard nine, ist eine auf neun Gruppen reduzierte C-Skala, die sich für die Normierung nicht-normalverteilter Daten eignet (Bühner, 2011). Zur Verdeutlichung: Der niedrigsten Staninegruppe (1) wurden die untersten $4 \%$

${ }^{1}$,Innovationen in der Ausbildung zum Lehramt für Berufskollegs am Studienstandort Münster" (vgl. Driesel-Lange et al., 2017), „Pilotprojekt Potentialanalyse“ (vgl. Driesel-Lange \& Kracke, 2017), Berliner Berufs- und Studienorientierungsstudie (vgl. Ohlemann et al., 2016). 
Tabelle 10.1 Instrumente zur Messung der Berufswahlkompetenz (Kaak et al., 2013)

\begin{tabular}{|c|c|c|c|}
\hline Skala ( $N$ Items) & $M$ & $S D$ & $\alpha$ \\
\hline Selbstwissen (9) & 3.15 & 0.52 & .83 \\
\hline Konzeptwissen (6) & 2.63 & 0.66 & .81 \\
\hline Bedingungswissen (7) & 2.84 & 0.72 & .87 \\
\hline Planung \& Entscheidung (4) & 2.66 & 0.72 & .67 \\
\hline Betroffenheit (8) & 3.38 & 0.58 & .88 \\
\hline Eigenverantwortung (6) & 3.06 & 0.64 & .85 \\
\hline Offenheit (7) & 2.55 & 0.61 & .79 \\
\hline Zuversicht (12) & 3.11 & 0.51 & .86 \\
\hline Exploration (9) & 2.16 & 0.71 & .89 \\
\hline Steuerung (7) & 2.91 & 0.61 & .81 \\
\hline Problemlösen (10) & 3.09 & 0.52 & .84 \\
\hline Stressmanagement (5) & 2.62 & 0.82 & .87 \\
\hline
\end{tabular}

Anmerkung. $M=$ Mittelwert, $S D=$ Standardabweichung, $\alpha=$ Cronbachs Alpha

Tabelle 10.2 Kernwert der Berufswahlkompetenz

\begin{tabular}{l|l|l|l|l|l}
\hline Skala & Min & Max & $M$ & $S D$ & $\alpha$ \\
\hline Kernwert der Berufswahlkompetenz & 1.56 & 3.96 & 2.84 & 0.41 & .86 \\
\hline
\end{tabular}

Anmerkung. Min $=$ Minimum, $\operatorname{Max}=$ Maximum

der Stichprobe, also jene mit dem niedrigsten BWK-Kernwert, zugeordnet. Die Jugendlichen mit dem höchsten BWK-Kernwert befinden sich entsprechend in der höchsten Gruppe, Stanine 9. Das beschriebene Prinzip der Staninezuordnung wird nochmals in Abbildung 10.1 ersichtlich. Ziel der Normierung anhand von Staninegruppen ist die Determinierung normierter Cut-Off-Werte für jede Gruppe. In einem weiteren Schritt wurden die neun Gruppen zu insgesamt drei Gruppen nochmals aggregiert, um mit drei Gruppen eine Anwendung zur schulischen Binnendifferenzierung organisatorisch zu vereinfachen. Mit Hilfe der Cut-OffWerte kann eine Zuteilung von weiteren Testpersonen zu den Gruppen erfolgen. Zudem kann eine zeitlich effiziente Einschätzung ihres übergreifenden Berufswahlkompetenzstands durch die Gruppenzugehörigkeit vorgenommen werden. Die Differenzierbarkeit zwischen den Gruppen hinsichtlich des BWK-Kernwerts wurde mittels des $95 \%$ Konfidenzintervalls des Mittelwerts untersucht. 


\subsection{Ergebnisse der Studie}

\section{Gruppen des Berufswahlkompetenzkernwerts (BWK-Kernwert)}

Aus den Mittelwerten der zwölf BWK-Facetten ergab sich ein BWK-Kernwert mit einer guten Reliabilität (Cronbachs $\alpha=.86$ ) und einem über dem statistischen Mittel liegenden Mittelwert von 2.84. Eine explorative Faktorenanalyse bestätigte zudem den Rückschluss auf eine Aggregation der Skalen. Die ursprünglich vierstufige Skala wurde in sich leicht gestaucht. Wie die Balken in Abbildung 10.1 zeigen, folgt die Verteilung der Stichprobe nach der Transformation in Staninewerte annähernd einer Normalverteilung.

Wie Abbildung 10.2 grafisch darstellt, bestehen zwischen den anhand des BWK-Kernwerts gebildeten Gruppen auch Unterschiede hinsichtlich der Entwicklungsstände der einzelnen BWK-Facetten. Auffallend ist, dass die neun Gruppen einem ähnlichen Kompetenzmuster folgen, bei dem die BWK-Facetten in ihrer durchschnittlichen Ausprägung jeweils in einem ähnlichen Verhältnis zueinanderstehen.

Das durchschnittliche Selbstwissen scheint in allen Gruppen jeweils weiter entwickelt zu sein als das Konzeptwissen. Bei den Handlungskompetenzen Exploration, Steuerung, Problemlösen und Stressmanagement lässt sich ein bogenförmiges Muster von der verhältnismäßig gering entwickelten Exploration, über die etwas stärker entwickelte Steuerung hin zur am stärksten entwickelten Problemlösekompetenz beobachten. Lediglich bei vier der zwölf BWK-Facetten (Betroffenheit, Offenheit, Exploration, Stressmanagement) lassen sich (leichte) Überschneidungen jeweils benachbarter Staninegruppen erkennen. Nach der Zusammenfassung der Staninegruppen in insgesamt drei Gruppen (Stanine 1-3= Gruppe 1) werden die parallelen Entwicklungsmuster, die sich vor allem in ihren Niveauausprägungen unterscheiden, noch deutlicher ersichtlich (siehe Abbildung 10.3). Die erste Hypothese, dass mittels des BWK-Kernwerts Gruppen mit ähnlichen Entwicklungsmustern gebildet werden können, lässt sich somit bestätigen.

\section{Differenzierbarkeiten der Gruppen auf den zwölf BWK-Facetten}

Für eine praktische Anwendung des BWK-Kernwerts im schulischen Kontext sind Gruppen mit trennscharfen Entwicklungsprofilen eine wichtige Voraussetzung, um anhand entsprechender Cut-Off-Werte eine Gruppenzuordnung weiterer Testpersonen vorzunehmen. Ziel wäre also eine möglichst klare Differenzierbarkeit zwischen den drei Gruppen auf allen zwölf BWK-Facetten, um vom BWK-Kernwert und den damit einhergehenden Gruppen mögliche Rückschlüsse auf die individuelle Entwicklung der einzelnen BWK-Facetten zur Feindiagnostik ziehen zu können. Zur 


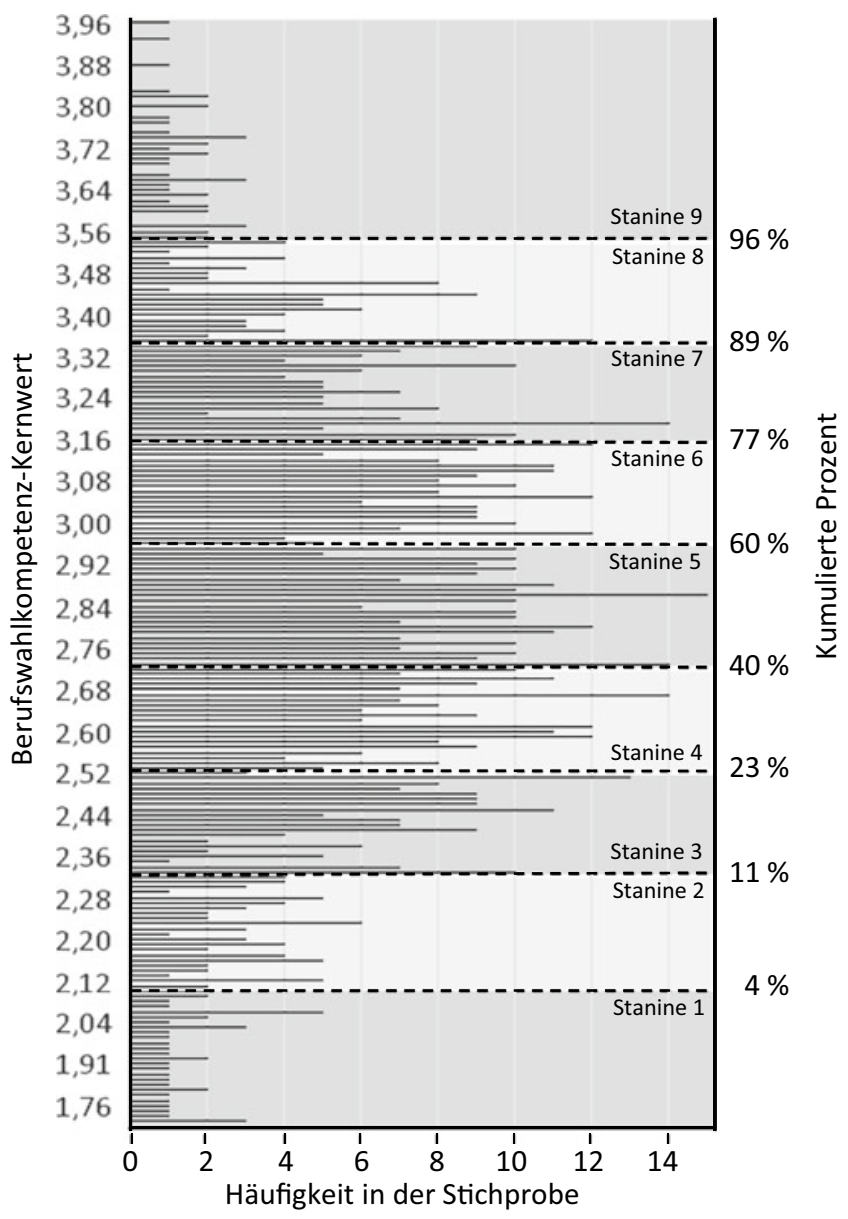

Abbildung 10.1 BWK-Kernwert: Verteilung der Stichprobe in Staninegruppen

Überprüfung dieser zweiten Hypothese wurde die Differenzierbarkeit der drei Gruppen auf BWK-Facettenebene anhand der $95 \%$ Konfidenzintervalle der Mittelwerte betrachtet und auf Überschneidungen zwischen den Gruppen überprüft. Liegt keine Überschneidung zwischen den Konfidenzintervallen der Gruppen vor, kann von rein differenzierbaren Facetten ausgegangen werden. Bei Überschneidungen einer oder mehrerer Gruppen handelt es sich um eine undifferenzierte Facette. Dazwischen 


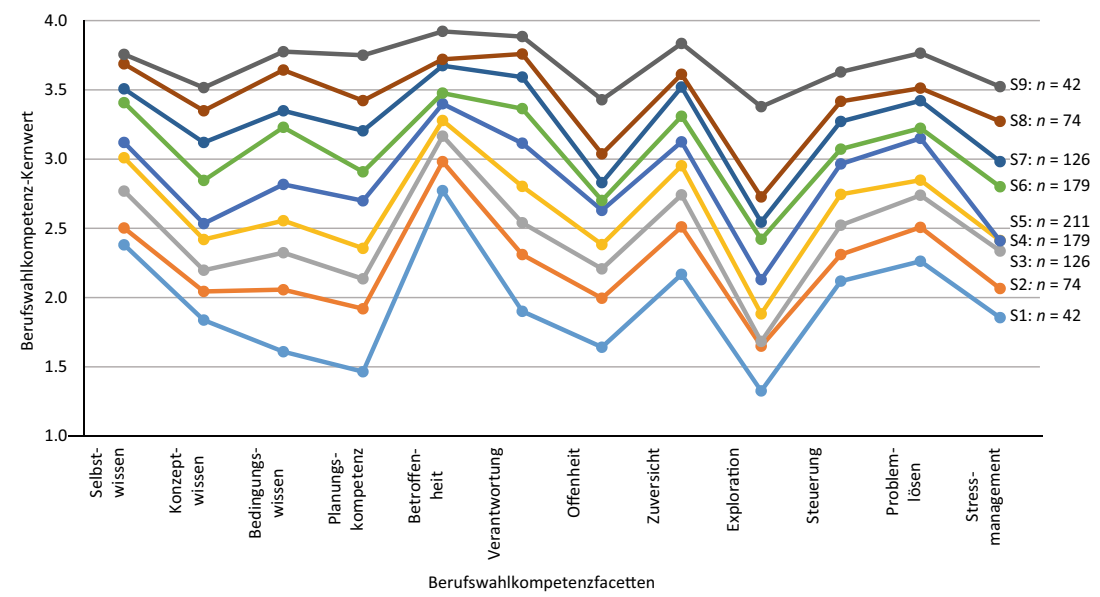

Abbildung 10.2 Übersicht der Berufswahlkompetenzfacetten der Staninegruppen (S1 S9).

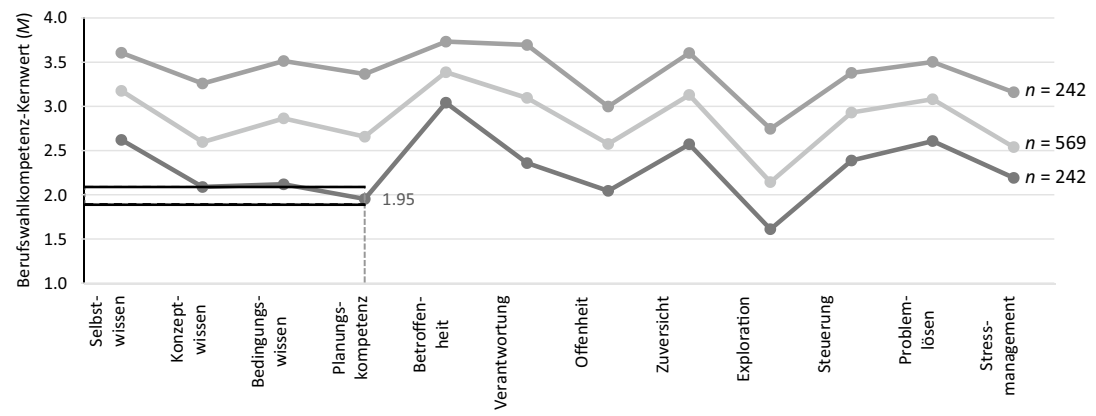

Abbildung 10.3 Rückschlüsse auf Entwicklungsstand einzelner Facetten: Beispiel Planung.

bestehen Abstufungen (vgl. Schurtz \& Artelt, 2014). Die Untersuchung der Konfidenzintervalle bestätigt die drei in erkennbaren Entwicklungsprofile. Beispielhaft anhand der Planungskompetenz zeigt die Tabelle 10.3 die Abgrenzung der drei Konfidenzintervalle: Schüler*innen der Gruppe 1 schätzten ihre Planungskompetenz mit einem Mittelwert von 1.95 im Vergleich zu den Gruppen 2 und 3 als am geringsten ein. Die Obergrenze des dazugehörigen Konfidenzintervalls liegt bei 2.02 und 
überschneidet sich nicht mit der Untergrenze des zweiten Konfidenzintervalls ( $C I$ = 2.61-2.70). Auch mit der dritten Gruppe bestehen keinerlei Überlappungen.

Der gleichen Analyse folgend lassen sich auf keiner BWK-Facette Überschneidungen zwischen den Konfidenzintervallen der drei Gruppen beobachten, sodass, wie mit Hypothese 2 angenommen, von einer reinen Differenzierbarkeit der Gruppen auf Ebene der BWK-Facetten ausgegangen werden kann.

\section{BWK-Kernwert: Rückschlüsse auf einzelne BWK-Facetten}

Am Beispiel der Planungskompetenz wird dargelegt, welche konkreten Rückschlüsse vom BWK-Kernwert auf seine einzelnen Facetten gezogen werden können (Hypothese 3). Testpersonen mit einem BWK-Kernwert zwischen 1.56 und 2.52 schätzen im Durchschnitt ihre individuelle Berufswahlkompetenzentwicklung im Vergleich zur hier betrachteten Stichprobe als gering entwickelt ein und werden anhand ihres BWK-Kernwerts der ersten Gruppe zugeordnet (Tabelle 10.3). Wie am $95 \%$ Konfidenzintervall des Mittelwerts abzulesen ist, liegt für diese Gruppe der Mittelwert für die Planungskompetenz mit einer 95 \%igen Wahrscheinlichkeit zwischen 1.88 und 2.02. Bei einer Person in Gruppe 1 könnte also von einer vergleichsweise gering ausgeprägten Planungskompetenz ausgegangen werden. Die dritte Hypothese, mögliche Rückschlüsse anhand des BWK-Kernwerts auf den Entwicklungsstand einzelner BWK-Facetten ziehen zu können, lässt sich damit bestätigen.

\subsection{Diskussion und Ausblick}

Zur Individualisierung beruflicher Orientierung könnte ein einfaches, standardisiertes Diagnostikverfahren zur Erfassung der Entwicklungsstände hilfreich sein, um in sich homogene Gruppen mit ähnlichen Bedarfen zu identifizieren. Für diese Gruppen mit ähnlichen Entwicklungsständen und Bedarfen könnte eine individualisierte(re) Zuteilung von BO-Maßnahmen organisatorisch vereinfacht bzw. überhaupt ermöglicht werden. Um Entwicklungsstände systematisch zu erheben, bedarf es eines Normwerts, der die BWK-Facetten einerseits zusammenfasst und andererseits Rückschlüsse auf sie zulässt.

Wie die vorgelegte Studie zeigt, lassen sich anhand des errechneten BWKKernwerts drei trennscharfe Gruppen mit ähnlichen Entwicklungsmustern für die zwölf BWK-Facetten identifizieren. Für die schulinterne Anwendung wichtig ist die Schlussfolgerung, dass anhand des BWK-Kernwerts Rückschlüsse auf den individuellen Entwicklungsstand auf Ebene der einzelnen Facetten möglich wären 


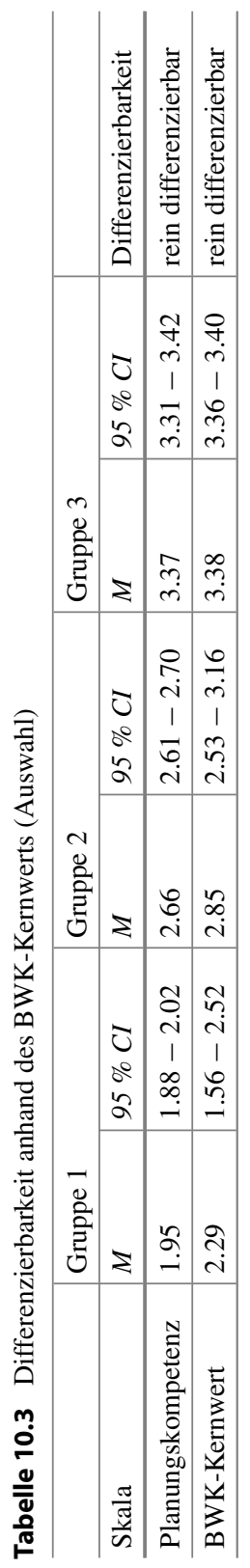


und unter Berücksichtigung der jeweiligen Berufswahlphase Förderbedarfe identifiziert werden könnten. Bei der vorgelegten Studie mit 1065 Teilnehmenden handelt es sich um eine explorative Untersuchung. Die berechneten Konfidenzintervalle können daher nicht ohne eine weitere Validierung anhand zusätzlicher Stichproben als normierte Cut-Off-Werte angesehen werden. Jedoch legitimieren die Ergebnisse die Weiterverfolgung des Prinzips eines zentralen Normwerts der Berufswahlkompetenz. Weiterführende Analysen in Bezug auf Schüler*innen unterschiedlicher sozioökonomischer Merkmale in unterschiedlichen Jahrgangsstufen an verschiedenen Schulformen sind notwendig, um mögliche Varianzen, wie sie auch bei Berufsinteressen und der Berufswahl in Abhängigkeit des Geschlechts zu beobachten sind, zu identifizieren (vgl. Faulstich-Wieland \& Scholand, 2017).

Ein Ziel wäre eine Normierung der Cut-Off-Werte der drei Gruppen für die diversen Zielgruppen, um eine standardisierte Nutzung des BWK-Kernwerts in Schulen zu ermöglichen. Denn unter dieser Prämisse bestätigter Normwerte, erscheint der BWK-Kernwert als diagnostisches Instrument zur Bestimmung berufswahlrelevanter Entwicklungsstände als vielversprechend. Mögliche Fehler in den Selbsteinschätzungen der Jugendlichen könnten in der Praxis durch eine sich anschließende kritische Reflexion der eigenen Einschätzungen im Gespräch mit einer berufsberatenden Fachkraft abgefedert werden. Ähnlich der Anwendung bereits etablierter Normwerte, wäre auch bei der Interpretation des BWK-Kernwerts zu beachten, dass es sich um einen statistischen Richtwert handelt. Eingebunden in ein breites Setting berufswahlrelevanter Unterstützung kann er jedoch als wichtige Orientierung dienen.

Open Access Dieses Kapitel wird unter der Creative Commons Namensnennung 4.0 International Lizenz (http://creativecommons.org/licenses/by/4.0/deed.de) veröffentlicht, welche die Nutzung, Vervielfältigung, Bearbeitung, Verbreitung und Wiedergabe in jeglichem Medium und Format erlaubt, sofern Sie den/die ursprünglichen Autor(en) und die Quelle ordnungsgemäß nennen, einen Link zur Creative Commons Lizenz beifügen und angeben, ob Änderungen vorgenommen wurden.

Die in diesem Kapitel enthaltenen Bilder und sonstiges Drittmaterial unterliegen ebenfalls der genannten Creative Commons Lizenz, sofern sich aus der Abbildungslegende nichts anderes ergibt. Sofern das betreffende Material nicht unter der genannten Creative Commons Lizenz steht und die betreffende Handlung nicht nach gesetzlichen Vorschriften erlaubt ist, ist für die oben aufgeführten Weiterverwendungen des Materials die Einwilligung des jeweiligen Rechteinhabers einzuholen.

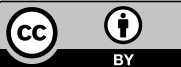

